\title{
Article
}

\section{Appendectomy and Non-Typhoidal Salmonella Infection: A Population-Based Matched Cohort Study}

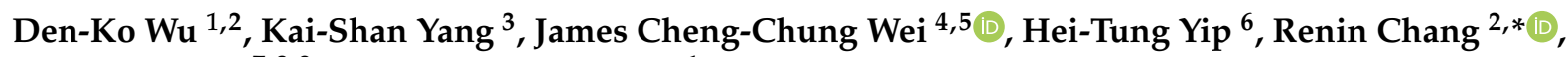 \\ Yao-Min Hung ${ }^{7,8,9, *}$ and Chih-Hsin Hung ${ }^{1, *}$
}

Citation: Wu, D.-K.; Yang, K.-S.; Wei, J.C.-C.; Yip, H.-T.; Chang, R.; Hung, Y.-M.; Hung, C.-H. Appendectomy and Non-Typhoidal Salmonella Infection: A Population-Based Matched Cohort Study. J. Clin. Med. 2021, 10, 1466. https://doi.org/ $10.3390 / \mathrm{jcm} 10071466$

Academic Editor: Andrew Day

Received: 12 February 2021

Accepted: 30 March 2021

Published: 2 April 2021

Publisher's Note: MDPI stays neutral with regard to jurisdictional claims in published maps and institutional affiliations.

Copyright: (C) 2021 by the authors. Licensee MDPI, Basel, Switzerland. This article is an open access article distributed under the terms and conditions of the Creative Commons Attribution (CC BY) license (https:// creativecommons.org/licenses/by/ $4.0 /)$.
1 Institute of Biotechnology and Chemical Engineering, I-Shou University, Kaohsiung 840, Taiwan; dkw3275@gmail.com

2 Department of Emergency Medicine, Kaohsiung Veterans General Hospital, Kaohsiung 813, Taiwan

3 School of Post-Baccalaureate Medicine, Kaohsiung Medical University, Kaohsiung 807, Taiwan; jenniferyang1993@gmail.com

4 Graduate Institute of Integrated Medicine, China Medical University, Taichung 404, Taiwan; wei3228@gmail.com

5 Division of Allergy, Immunology and Rheumatology, Institute of Medicine, Chung Shan Medical University, Taichung 402, Taiwan

6 Management Office for Health Data, China Medical University Hospital, Taichung 404, Taiwan; fionyip0i0@gmail.com

7 Department of Internal Medicine, Kaohsiung Municipal United Hospital, Kaohsiung 804, Taiwan

8 College of Health and Nursing, Meiho University, Pingtung 912, Taiwan

9 Department of Internal Medicine, Institute of Medicine, Chung Shan Medical University, Taichung 402, Taiwan

* Correspondence: rhapsody1881@gmail.com (R.C.); ymhung1@gmail.com (Y.-M.H.); chhung@isu.edu.tw (C.-H.H.); Tel.: +886-73422121 (R.C.); +886-75552565 (Y.-M.H.); +886-76577711 (ext. 3414) (C.-H.H.)

Abstract: The potential association between appendectomy and non-typhoidal Salmonella (NTS) infection has not been elucidated. We hypothesized that appendectomy may be associated with gut vulnerability to NTS. The data were retrospectively collected from the Taiwan National Health Insurance Research Database to describe the incidence rates of NTS infection requiring hospital admission among patients with and without an appendectomy. A total of 208,585 individuals aged $\geq 18$ years with an appendectomy were enrolled from January 2000 to December 2012, and compared with a control group of 208,585 individuals who had never received an appendectomy matched by propensity score (1:1) by index year, age, sex, occupation, and comorbidities. An appendectomy was defined by the International Classification of Diseases, Ninth Revision, Clinical Modification Procedure Codes. The main outcome was patients who were hospitalized for NTS. Cox proportional hazards models were applied to estimate the hazard ratios (HRs) and 95\% confidence intervals (CIs). Two sensitivity analyses were conducted for cross-validation. Of the 417,170 participants (215,221 (51.6\%) male), 208,585 individuals (50.0\%) had an appendectomy, and 112 individuals developed NTS infection requiring hospitalization. In the fully adjusted multivariable Cox proportional hazards regression model, the appendectomy group had an increased risk of NTS infection (adjusted HR (aHR), 1.61; 95\% CI, 1.20-2.17). Females and individuals aged 18 to 30 years with a history of appendectomy had a statistically higher risk of NTS than the control group (aHR, 1.92; 95\% CI, 1.26-2.93 and aHR, $2.67 ; 95 \%$ CI, 1.41-5.07). In this study, appendectomy was positively associated with subsequent hospitalization for NTS. The mechanism behind this association remains uncertain and needs further studies to clarify the interactions between appendectomy and NTS.

Keywords: appendectomy; non-typhoidal Salmonella infection; NTS; cohort study; National Health Insurance Research Database 


\section{Introduction}

Appendectomy is one of the most-commonly performed surgical procedures in the world. A recent meta-analysis of the incidence of appendectomy in Northern America was 100 per 100,000 person years [1], while it was 107.76 in Taiwan [2]. Studies have shown that the appendix may be an important component of human immune function [3,4]. Absence of an appendix has been mentioned in relation to recurrent infection with Clostridium difficile [5]. Recently, a study that enrolled patients who underwent incidental prophylactic appendectomy during 2004-2008 showed profound and long-term dysbiosis in these patients, sometimes for years [6]. Reduced microbial diversity may reflect the severity of the disease in critically ill patients and be associated with mortality [7]. Appendectomy might disrupt the immune function and studies have observed the relationship between antecedent appendix removal and the risk of pulmonary tuberculosis and sepsis [8,9]. Global non-typhoidal Salmonella (NTS) infection occurs in millions of people annually [10-14]. NTS may cause severe invasive bacteremia or disseminated disease $[15,16]$. The numbers of host risk factors predispose individuals to NTS $[17,18]$. These risk factors include the extremes of age [19], diabetes [19], malignancy [20], rheumatologic disease [19,21], use of immunomodulatory drugs [18], transplantation [22], and HIV infection [17,23]. About half a century ago, gastrectomy had been shown to be associated with an increased risk of subsequent NTS infection due to achlorhydria, rapid food emptying and altered intestinal flora [24]. Nowadays, it is widely accepted that when the bacterial population in the gastrointestinal tract is unstable, NTS is more likely to take advantage of the situation and invade the gastrointestinal tract [25]; on the other hand, appendectomy might cause long-term disturbance of the microbiome [6]. We hypothesized that patients who experienced removal of the appendix were susceptible to NTS. This population-based propensity score-matched (PSM) cohort study was conducted to examine the impact of appendectomy on subsequent NTS infections requiring hospital admission.

\section{Materials and Methods}

\subsection{Data Source}

Since 1995, more than $99 \%$ of the Taiwan population have been insured through a single-payer National Health Insurance program launched by the government. The medical claims contribute to the National Health Insurance Research Database (NHIRD). Previous studies demonstrated the high validity of data derived from the NHIRD [26]. This study used the hospitalization dataset, which records the disease diagnosis and procedure of therapy received during the admission. The diagnostic codes of the claims are recorded according to the International Classification of Diseases, 9th Revision, Clinical Modification (ICD-9-CM).

\subsection{Standard Protocol Approvals, Registrations, and Patient Consents}

The Research Ethics Committee of China Medical University and Hospital in Taiwan (CMUH104-REC2-115(AR-4)) approved this study. As the data used consisted of the de-identified secondary data set released for research purposes and were analyzed anonymously, the need for informed consent was waived.

\subsection{Study Subjects}

An appendectomy was defined according to the ICD-9-CM procedure code 47 . The appendectomy group consisted of 208,585 individuals ages 18 and over with a newly received appendectomy from 1 January 2000, through 31 December 2012; individuals who received an appendectomy from 1997 to 1999 were excluded. To minimize confounding from other alimentary surgical procedures, individuals who received a gastrectomy (ICD9-CM procedure code 43.5-43.9), cholecystectomy (ICD-9-CM procedure code 51.2), or intestinal or large bowel resection (ICD-9-CM procedure code 45.6-45.9) before the index date or underwent multiple concurrent procedures at the time of appendectomy were 
excluded. Patients diagnosed with cancer (ICD-9-CM code 140-208) before the index date were also excluded.

As proton pump inhibitors provide a favorable environment for NTS, patients with peptic ulcer disease (a proxy for proton pump inhibitors) before the index date were excluded. Individuals with hospitalized NTS within one month after the index date were also excluded to avoid confounding by the possible effect of perioperative antibiotics. The first date of hospitalization for appendectomy was the index date, and this date was assigned to the accordant matched controls (defined as the first healthcare use occurring in the index year) with the same criteria. Patients having a history of NTS are at risk for recurrent NTS, so those patients were also excluded.

Finally, 208,585 patients with appendectomy without a medical history of NTS before the index date (traced back from 1997 through 1999) were included. To minimize surveillance bias, these exposed participants were compared with the 208,585 sex-, age-, index date-and comorbidity-matched individuals in the non-appendectomy group by propensity score matching (PSM) from the same inpatient dataset. We performed a rematch by greedy algorithm. For each study case with appendectomy, the corresponding comparison case without appendectomy was selected based on the closest propensity score. Propensity scores were calculated using a logistic regression model to calculate the probability of appendectomy assignment and included the following baseline variables: sex, age, occupation, and year of index date. The comorbidities analyzed in the study included hypertension (ICD-9-CM code 401-405), diabetes (ICD-9-CM code 250), hyperlipidemia (ICD-9-CM code 272), coronary artery disease (CAD) (ICD-9-CM code 410-414), cerebrovascular disease (CVD) (ICD-9-CM code 430-438), chronic kidney disease (CKD) (ICD-9-CM code 585), chronic obstructive pulmonary disease (COPD) (ICD-9-CM code 491, 492, 496), human immunodeficiency virus (HIV) (ICD-9-CM code 042), liver cirrhosis (ICD-9-CM code 571.5), and systemic lupus erythematosus (SLE) (ICD-9-CM code 710.0).

Each case in the study and control groups were followed from individual index date until an event (hospitalization for NTS), withdrawal from the NHI program or December 2013. We adopted the PSM method to account for a similar distribution of baseline characteristics between both groups.

\subsection{Identification of Main Outcome}

The outcome was patients with NTS recorded in the hospitalization dataset, a subset of the NHIRD; the incidence of new-onset NTS depends upon the administrative ICD coding of 003.xx [11]. The physician responsible for the patient must make the diagnosis using the appropriate ICD code based on careful evaluation and examination, including analysis of stool and/or blood cultures. The coding system is considered validated as the government periodically audits claims for payment purposes. The fine for fraud is 100 times the amount of the fraudulent claims collected from the NHI Bureau. To control the possible bias due to perioperative antibiotics, individuals experienced NTS within one month of the index date were excluded.

\subsection{Negative Exposure Control Analysis}

Negative control has been used to detect unmeasured confounding. Diverticulitis was selected as an alternative exposure (ICD-9-CM code 562.x), and based on review of current pathophysiological mechanisms, it was not associated with subsequent NTS. Therefore, any association between diverticulitis and subsequent NTS may hint at the presence of unmeasured confounding factors.

\subsection{Statistical Analysis}

The first record of each participant hospitalized for NTS was used to calculate the risk of NTS. The density of NTS events per 10,000 person-years was calculated in both groups. We used PSM to control for sampling bias. The propensity score presented an individual's probability of developing NTS, and the score was determined by a multivariable logistic 
regression model. The difference of the baseline characteristics between the study and the comparison group were compared by the standardized mean difference (SMD). A SMD of 0.1 or less indicates a negligible difference between the two groups.

We estimated the crude hazard ratio (HR) and 95\% confidence interval (CI) using the univariable Cox proportional hazard model. Variables found to be statistically significant in the univariable model were further examined in the multivariable model. The multivariable Cox proportional hazard model was used to estimate the adjusted HR (aHR), including hypertension, diabetes, CAD, CVD, CKD, COPD, HIV, liver cirrhosis, and SLE. The Kaplan-Meier method was adopted to obtain the cumulative incidence of NTS in the two groups. The log-rank test was utilized to compare the differences between the two groups. All statistical tests were two-sided, and $p$ values of 0.05 or less were considered statistically significant.

In the main model, we excluded patients with PUD (a proxy to minimize the effect of proton pump inhibitors utilization) [11,27] before the index date and patients who had NTS within one month of the index date (a proxy to minimize the effect of perioperative antibiotic utilization). To validate the findings in the main model, several post hoc sensitivity analyses by different definitions of enrollment (model 2 to 5) were conducted. Because the effects of antibiotic treatment might be longer lasting than 30 days; in model 2, we excluded patients having NTS infection occurred within 90 days of the index date. In model 3 , because antibiotic utilization is likely a significant confounder, and with no prescription information in the hospitalization dataset, we excluded patients having bacterial infection within 6 months before the index date (a proxy to minimize the effect of antibiotic utilization on the participants; ICD-9 codes of bacterial infection are 001-005, 008.1-008.5, 020-027, 030-041, 076, 320, 420.9, 421.0, 422.92, 481-483, 511.1, 522.4-522.7, 523.3-523.5, 527.3, 528.3, 566, 567.0-567.2, 569.5, 572.0, 590, 595.89, 595.9, 597.0, 599.0, 614-616, 680-686, 785.52). In model 4, PUD and other comorbidities related to immunocompromise that conferred increased risk of NTS infection were excluded before the index date. In model 5, PUD and other comorbidities related to immunocompromise that confer increased risk of NTS infection were not excluded and adjusted as covariates in regression analysis.

\section{Results}

\subsection{Patient Characteristics}

Of 417,170 participants $(215,221(51.6 \%)$ male) aged 18 years and older, 208,585 individuals (50\%) had experienced appendectomy (107,823 male (52\%)) and 112 individuals $0.05 \%$ developed hospitalized NTS. The 208,585 individuals who did not have appendectomy $(107,398$ men $(51 \%))$ were matched by age, sex, and comorbidities (Table 1). PSM resulted in 208,585 matched individuals in each group. In the study group and comparison group, the baseline characteristics were well balanced. The mean (SD) age was 38.8 (15.2) years in the study group and 40.8 (16.7) years in the control group. The median (SD) follow-up times were 7.29 (3.87) years in the study group and 6.73 (3.54) years in the control group. Individuals in the study group, compared with those in the control group, had similar proportions of occupation and comorbidities but a lower proportion of hypertension (13,982 individuals (7\%) vs. 26,344 individuals (13\%); SMD, 0.20$)$, diabetes (7646 individuals (3.7\%) vs. 12,891 individuals $(6.2 \%)$; SMD, 0.12$)$, and cerebrovascular disease (3189 individuals (1.5\%) vs. 1492 individuals (3.1\%); SMD, 0.11). The mean (SD) hospital stay for appendectomy was 5.65 (42.9). 
Table 1. Baseline Patient Characteristics.

\begin{tabular}{|c|c|c|c|c|c|}
\hline \multirow[b]{3}{*}{ Variables } & \multicolumn{2}{|c|}{ Non-Appendectomy } & \multicolumn{2}{|c|}{ Appendectomy } & \multirow[b]{3}{*}{ SMD } \\
\hline & \multicolumn{2}{|c|}{$(\mathrm{N}=208,585)$} & \multicolumn{2}{|c|}{$(N=208,585)$} & \\
\hline & $\mathrm{n}$ & $\%$ & $\mathrm{n}$ & $\%$ & \\
\hline Gender & & & & & 0.004 \\
\hline Female & 101,187 & $49 \%$ & 100,762 & $48 \%$ & \\
\hline Male & 107,398 & $51 \%$ & 107,823 & $52 \%$ & \\
\hline \multicolumn{6}{|l|}{ Age group } \\
\hline $18-30$ & 67,712 & $32 \%$ & 73,158 & $35 \%$ & 0.06 \\
\hline $31-40$ & 48,032 & $23 \%$ & 51,535 & $25 \%$ & 0.04 \\
\hline $41-50$ & 38,410 & $18 \%$ & 38,985 & $18.5 \%$ & 0.007 \\
\hline $51-60$ & 23,946 & $12 \%$ & 22,957 & $11 \%$ & 0.02 \\
\hline $61-70$ & 14,661 & $7 \%$ & 11,934 & $5.7 \%$ & 0.05 \\
\hline $71-80$ & 11,166 & $5.4 \%$ & 7381 & $3.5 \%$ & 0.09 \\
\hline $81-100$ & 4658 & $2.2 \%$ & 2635 & $1.3 \%$ & 0.07 \\
\hline mean, (SD) & 40.8 & $(16.7)$ & 38.8 & $(15.2)$ & 0.13 \\
\hline \multicolumn{6}{|l|}{ Occupation } \\
\hline white-collar worker & 109,333 & $52 \%$ & 114,108 & $55 \%$ & 0.046 \\
\hline blue-collar worker & 47,233 & $23 \%$ & 48,197 & $23 \%$ & 0.011 \\
\hline farmer & 4268 & $2 \%$ & 4053 & $2 \%$ & 0.007 \\
\hline fisher & 25,437 & $12 \%$ & 22,041 & $11 \%$ & 0.05 \\
\hline others & 22,314 & $11 \%$ & 20,186 & $10 \%$ & 0.03 \\
\hline \multicolumn{6}{|l|}{ Comorbidities } \\
\hline hypertension & 26,344 & $13 \%$ & 13,982 & $7 \%$ & 0.20 \\
\hline diabetes & 12,891 & $6.2 \%$ & 7646 & $3.7 \%$ & 0.12 \\
\hline hyperlipidemia & 5811 & $2.8 \%$ & 3185 & $1.5 \%$ & 0.09 \\
\hline CAD & 8518 & $4.1 \%$ & 3908 & $1.9 \%$ & 0.13 \\
\hline CVD & 6512 & $3.1 \%$ & 3189 & $1.5 \%$ & 0.11 \\
\hline CKD & 1492 & $0.7 \%$ & 762 & $0.4 \%$ & 0.05 \\
\hline COPD & 3235 & $1.6 \%$ & 1532 & $0.7 \%$ & 0.08 \\
\hline HIV & 105 & $0.1 \%$ & 93 & $0.0 \%$ & 0.003 \\
\hline Liver cirrhosis & 1036 & $0.5 \%$ & 551 & $0.3 \%$ & 0.04 \\
\hline SLE & 506 & $0.2 \%$ & 233 & $0.1 \%$ & 0.03 \\
\hline
\end{tabular}

Abbreviations: CAD: coronary artery disease; CVD: cerebrovascular disease; CKD: chronic kidney disease; COPD: chronic obstructive pulmonary disease; HIV: human immunodeficiency virus; SLE: systemic lupus erythematosus.

\subsection{Outcomes}

Table 2 shows the results of the univariable and multivariable Cox regression analysis, in which the incidence rate of NTS after appendectomy was 0.74 per 10,000 person-years and that in the comparison group was 0.55 per 10,000 person-years. There were 77 events of hospitalized NTS without appendectomy, and 112 events of hospitalized NTS after undergoing appendectomy. The individuals who had histories of appendectomy were more likely to develop NTS (unadjusted HR, 1.35; 95\% CI, 1.01-1.8). The multivariable Cox regression analysis showed a positive association between appendectomy and new-onset hospitalized NTS. After adjusting for demographics, occupation, and comorbidities (except hyperlipidemia) at the baseline, individuals with appendectomy had a $61 \%$ increased risk of developing hospitalized NTS than subjects without appendectomy, with an adjusted HR of 1.61 (95\% CI, 1.20-2.17). Table 2 also presents the relevant risk factors for NTS infection, and these were age 61-70, 71-80. The 81-100 group with aHR 2.62 (95\% CI, 1.57-4.37), 2.51 (95\% CI, 1.34-4.60), 4.50 (95\% CI, 2.16-9.38), diabetes (aHR, 1.75), COPD (aHR, 2.65), liver cirrhosis (aHR 4.48), HIV (aHR, 22.8), and SLE (aHR, 23.3). Thus, this study proposes appendectomy as a potential novel risk for subsequent NTS infection requiring hospitalization. 
Table 2. Hospitalized non-typhoidal Salmonella (NTS) infection incidence rate and risk factors.

\begin{tabular}{|c|c|c|c|c|c|c|c|}
\hline \multirow[b]{2}{*}{ Variables } & \multirow[b]{2}{*}{ Events } & \multirow[b]{2}{*}{ PY } & \multirow[b]{2}{*}{ IR } & \multicolumn{2}{|c|}{ Crude Analysis } & \multicolumn{2}{|c|}{ Adjusted Analysis ${ }^{\dagger}$} \\
\hline & & & & HR & $95 \% \mathrm{CI}$ & HR & $95 \%$ CI \\
\hline \multicolumn{8}{|l|}{ Appendectomy } \\
\hline No & 77 & $1,402,999$ & 0.55 & 1.00 & (reference) & 1.00 & (reference) \\
\hline Yes & 112 & $1,521,600$ & 0.74 & 1.35 & $(1.01,1.8)^{*}$ & 1.61 & $(1.20,2.17)^{* *}$ \\
\hline \multicolumn{8}{|l|}{ Gender } \\
\hline Female & 96 & $1,426,675$ & 0.67 & 1.00 & (reference) & & \\
\hline Male & 93 & $1,497,924$ & 0.62 & 0.92 & $(0.69,1.23)$ & & \\
\hline \multicolumn{8}{|l|}{ Age group } \\
\hline $18-30$ & 49 & $1,056,867$ & 0.46 & 1.00 & (reference) & 1.00 & (reference) \\
\hline $31-40$ & 36 & 706,585 & 0.51 & 1.10 & $(0.72,1.69)$ & 1.05 & $(0.68,1.61)$ \\
\hline $41-50$ & 31 & 550,161 & 0.56 & 1.22 & $(0.78,1.91)$ & 1.15 & $(0.73,1.80)$ \\
\hline $51-60$ & 15 & 300,584 & 0.49 & 1.09 & $(0.61,1.95)$ & 0.93 & $(0.52,1.68)$ \\
\hline $61-70$ & 27 & 168,690 & 1.60 & 3.49 & $(2.18,5.59)^{* * *}$ & 2.62 & $(1.57,4.37)^{* * *}$ \\
\hline $71-80$ & 19 & 109,128 & 1.74 & 3.84 & $(2.25,6.52)^{* * *}$ & 2.51 & $(1.37,4.60)^{* * *}$ \\
\hline $81-100$ & 12 & 32,584 & 3.68 & 8.16 & $(4.32,15.4)^{* * *}$ & 4.50 & $(2.16,9.38)^{* * *}$ \\
\hline \multicolumn{8}{|l|}{ Occupation } \\
\hline white-collar worker & 102 & $1,568,790$ & 0.65 & 1.00 & (reference) & & \\
\hline blue-collar worker & 47 & 668,814 & 0.70 & 1.08 & $(0.77,1.53)$ & & \\
\hline farmer & 3 & 59,873 & 0.50 & 0.77 & $(0.24,2.43)$ & & \\
\hline fisher & 25 & 331,922 & 0.75 & 1.16 & $(0.75,1.80)$ & & \\
\hline others & 12 & 295,200 & 0.41 & 0.63 & $(0.34,1.14)$ & & \\
\hline \multicolumn{8}{|l|}{ Comorbidities } \\
\hline \multicolumn{8}{|l|}{ hypertension } \\
\hline No & 152 & $2,714,893$ & 0.56 & 1.00 & (reference) & 1.00 & (reference) \\
\hline Yes & 37 & 209,706 & 1.76 & 3.21 & $(2.24,4.62)^{* * *}$ & 1.12 & $(0.67,1.85)$ \\
\hline \multicolumn{8}{|l|}{ diabetes } \\
\hline No & 166 & $2,813,306$ & 0.59 & 1.00 & (reference) & 1.00 & (reference) \\
\hline Yes & 23 & 111,293 & 2.07 & 3.53 & $(2.28,5.48)^{* * *}$ & 1.75 & $(1.06,2.88) *$ \\
\hline \multicolumn{8}{|l|}{ hyperlipidemia } \\
\hline No & 183 & $2,875,027$ & 0.64 & 1.00 & (reference) & & \\
\hline Yes & 6 & 49,573 & 1.21 & 1.90 & $(0.84,4.30)$ & & \\
\hline \multicolumn{8}{|l|}{ CAD } \\
\hline No & 171 & $2,857,167$ & 0.60 & 1.00 & (reference) & 1.00 & (reference) \\
\hline Yes & 18 & 67,432 & 2.67 & 4.50 & $(2.76,7.32)^{* * *}$ & 1.59 & $(0.89,2.84)$ \\
\hline \multicolumn{8}{|l|}{ CVD } \\
\hline No & 176 & $2,874,382$ & 0.61 & 1.00 & (reference) & 1.00 & (reference) \\
\hline Yes & 13 & 50,218 & 2.59 & 4.25 & $(2.42,7.48)^{* * *}$ & 1.53 & $(0.80,2.90)$ \\
\hline \multicolumn{8}{|l|}{ CKD } \\
\hline No & 186 & $2,914,193$ & 0.64 & 1.00 & (reference) & 1.00 & (reference) \\
\hline Yes & 3 & 10,406 & 2.88 & 4.50 & $(1.44,14.1)^{* *}$ & 1.52 & $(0.46,4.89)$ \\
\hline
\end{tabular}


Table 2. Cont.

\begin{tabular}{|c|c|c|c|c|c|c|c|}
\hline \multirow[b]{2}{*}{ Variables } & \multirow[b]{2}{*}{ Events } & \multirow[b]{2}{*}{ PY } & \multirow[b]{2}{*}{ IR } & \multicolumn{2}{|c|}{ Crude Analysis } & \multicolumn{2}{|c|}{ Adjusted Analysis ${ }^{\dagger}$} \\
\hline & & & & HR & $95 \% \mathrm{CI}$ & HR & $95 \%$ CI \\
\hline \multicolumn{8}{|l|}{ COPD } \\
\hline No & 178 & $2,899,817$ & 0.61 & 1.00 & (reference) & 1.00 & (reference) \\
\hline Yes & 11 & 24,782 & 4.44 & 7.23 & $(3.93,13.31)^{* * *}$ & 2.65 & $(1.36,5.16)^{* *}$ \\
\hline \multicolumn{8}{|l|}{ HIV } \\
\hline No & 188 & $2,923,704$ & 0.64 & 1.00 & (reference) & 1.00 & (reference) \\
\hline Yes & 1 & 895 & 11.2 & 17.24 & $(2.41,123.1)^{* *}$ & 22.8 & $(3.18,163.81)^{* *}$ \\
\hline \multicolumn{8}{|c|}{ Liver cirrhosis } \\
\hline No & 185 & $2,916,568$ & 0.63 & 1.00 & (reference) & 1.00 & (reference) \\
\hline Yes & 4 & 8031 & 4.98 & 7.88 & $(2.92,21.23)^{* * *}$ & 4.48 & $(1.64,12.25)^{* *}$ \\
\hline \multicolumn{8}{|l|}{ SLE } \\
\hline No & 183 & $2,919,707$ & 0.63 & 1.00 & (reference) & 1.00 & (reference) \\
\hline Yes & 6 & 4893 & 12.3 & 19.3 & $(8.58,43.63)^{* * *}$ & 23.3 & $\underset{* * *}{(10.20,53.06)}$ \\
\hline
\end{tabular}

Abbreviations: CAD: coronary artery disease; CVD: cerebrovascular disease; CKD: chronic kidney disease; COPD: chronic obstructive pulmonary disease; HIV: human immunodeficiency virus; SLE: systemic lupus erythematosus; ${ }^{*}: p$-value $<0.05 ;{ }^{* *}: p$-value $<0.01 ;{ }^{* * *}$ : $p$-value $<0.001 ; \mathrm{CI}$, confidence interval; HR, hazard ratio; PY: person-years; IR, incidence rate per 10,000 person-years. ${ }^{\dagger}$ : adjusted for age and all comorbidities except hyperlipidemia.

\subsection{Sensitivity Analyses}

Table 3 provides five models to examine the stability of HR of hospitalized NTS infection with the different definitions of appendectomy exposure and events of main outcome. In the subset of NHIRD (hospitalization dataset analyzed in this study), there was no prescription information to identify the length of antibiotic treatment. We developed 1,2 , and 3 to minimize the effect of antibiotic utility. The wash-out period in model 2 was up to 90 days. The aHRs were 1.61 (95\% CI 1.20 to 2.17), 1.58 (95\% CI 1.17 to 2.13 ) and 1.61 (95\% CI 1.20 to 2.16) in models 1, 2 and 3. Furthermore, in model 4, we excluded immunocompromised cases potentially prone to have NTS infection and the aHR was 1.71 (95\% CI 1.26 to 2.33) of NTS infection for appendectomy exposure. In model 5, PUD was included into regression analysis and the aHR was 1.24 (95\% CI 1.02 to 1.52$)$.

Table 3. Sensitivity Analyses.

\begin{tabular}{cc}
\hline & Compared to Patients without Appendectomy \\
\hline & aHR (95\% CI) \\
\hline Model 1 (Main model) & $1.61(1.20,2.17)^{* *}$ \\
Model 2 & $1.58(1.17,2.13)^{* *}$ \\
Model 3 & $1.61(1.20,2.16)^{* * *}$ \\
Model 4 & $1.71(1.26,2.33)^{* * *}$ \\
Model 5 & $1.24(1.02,1.52)^{*}$
\end{tabular}

Model 1: excluded patients with NTS occurred $<1$ month after index date or PUD before index date or adjusted demographics and all comorbidities except hyperlipidemia in Table 1. Model 2: excluded patients with NTS occurred $<3$ months after index date or PUD before index date. Model 3: excluded patients with recent bacterial infection within 6 months before the index date. Model 4: excluded patients with HIV, liver cirrhosis and SLE before the index date. Model 5: included patients with PUD into the main model analysis. aHR: adjusted hazard ratio; CI: confidence interval; * $p$-value $<0.05 ;{ }^{* *}$ : $p$-value $<0.01$; $^{* * *}$ : $p$-value $<0.001$.

\subsection{Subgroup Analysis}

Table 4 reveals the risk of NTS infection requiring hospitalization in different subgroups. In the age subgroup analysis, in comparison to the age-matched non-appendectomy 
controls, individuals aged 18 to 30 years with appendectomy had a significantly higher risk of subsequent hospitalized NTS (adjusted HR, 2.67; 95\% CI, 1.41-5.07; $p<0.01$ ). Prior history of appendectomy in patients age 51-60, 61-70, 71-80, and 81-100 have adjusted HR of 2.68 (95\% CI, 0.85-8.47), 1.12 (95\% CI, 0.51-2.45), 0.90 (95\% CI, 0.34-2.35), and 0.34 (95\% CI, $0.07-1.63$ ) respectively. The interaction for the age subgroup was not significant ( $p$ value for interaction $=0.10$ ). In the sex subgroup analysis, females with a history of appendectomy had an increased risk of NTS infection compared with females without appendectomy (adjusted HR, 1.92; 95\% CI, 1.26-2.93; $p<0.01$ ), while there was no significant association between appendectomy and risk of NTS infection for male patients. However, the $p$ value for interaction was not significant (0.82). In the occupation subgroup analysis, compared to matched participants without appendectomy, individuals with a white-collar occupation had a significantly increased risk of subsequent hospitalized NTS (adjusted HR, 1.76, 95\% CI, 1.17-2.65; $p$ <.01). In the comorbidity-subgroup analysis, in general, compared with matched patients without appendectomy, the risk of hospitalized NTS infection became nonsignificant whenever any one of the comorbidities presented. Appendectomy appeared to have a higher association in the relatively healthy participants in the study (e.g., within all participants without hypertension, subjects in the appendectomy group were at higher risk of NTS infection in comparison to participants without appendectomy; aHR, 1.77; 95\% CI, 1.26-2.47; within all participants without diabetes, subjects in the appendectomy group were at higher risk of NTS in comparison to participants without appendectomy; aHR, 1.72; 95\% CI, 1.25-2.37; and within all participants without SLE, subjects in the appendectomy group were at higher risk of NTS infection in comparison to participants without appendectomy, aHR, 1.67; 95\% CI, 1.24-2.26).

Table 5 displays our analysis stratified by the follow-up years. In the first six months, the relative risk of hospitalized NTS compared with the subjects without appendectomy was 1.83 (95\% CI, 0.74-4.53). During the follow up of six months to one year after appendectomy, the relative risk of having hospitalized NTS was 0.68 (95\% CI, 0.25-1.89). After $>1$ year of follow up, the adjusted HR was 1.74 (95\% CI, 1.25-2.43). 
Table 4. Subgroup analysis.

\begin{tabular}{|c|c|c|c|c|c|c|c|c|c|c|c|}
\hline & \multicolumn{6}{|c|}{ Appendectomy } & \multicolumn{2}{|c|}{ Crude Analysis } & \multicolumn{2}{|c|}{ Adjusted Analysis ${ }^{+}$} & \multirow[b]{2}{*}{$p$ for Interaction } \\
\hline & & No & & & Yes & & & & & & \\
\hline Variables & Events & PY & IR & Events & PY & IR & HR & $95 \% \mathrm{CI}$ & HR & $95 \% \mathrm{CI}$ & \\
\hline Gender & & & & & & & & & & & 0.82 \\
\hline Female & 36 & 683,410 & 0.53 & 60 & 743,265 & 0.81 & 1.56 & $(1.03,2.36) *$ & 1.92 & $(1.26,2.93)^{* *}$ & \\
\hline Male & 41 & 719,589 & 0.57 & 52 & 778,335 & 0.67 & 1.16 & $(0.77,1.75)$ & 1.33 & $(0.88,2.0)$ & \\
\hline Age group & & & & & & & & & & & 0.10 \\
\hline $31-40$ & 13 & 328,638 & 0.4 & 23 & 377,947 & 0.61 & 1.57 & $(0.79,3.10)$ & 1.71 & $(0.86,3.40)$ & \\
\hline $41-50$ & 10 & 260,773 & 0.38 & 21 & 289,389 & 0.73 & 1.91 & $(0.90,4.06)$ & 2.05 & $(0.96,4.38)$ & \\
\hline $51-60$ & 4 & 147,585 & 0.27 & 11 & 153,000 & 0.72 & 2.62 & $(0.83,8.24)$ & 2.68 & $(0.85,8.47)$ & \\
\hline $61-70$ & 15 & 88,397 & 1.70 & 12 & 80,294 & 1.49 & 0.86 & $(0.40,1.84)$ & 1.12 & $(0.51,2.45)$ & \\
\hline $71-80$ & 12 & 64,375 & 1.86 & 7 & 44,753 & 1.56 & 0.82 & $(0.32,2.09)$ & 0.90 & $(0.34,2.35)$ & \\
\hline $81-100$ & 10 & 20,672 & 4.84 & 2 & 11,912 & 1.68 & 0.35 & $(0.08,1.60)$ & 0.34 & $(0.07,1.63)$ & \\
\hline Occupation & & & & & & & & & & & 0.63 \\
\hline white-collar worker & 38 & 739,180 & 0.51 & 64 & 829,610 & 0.77 & 1.52 & $(1.02,2.27) *$ & 1.76 & $(1.17,2.65)^{* *}$ & \\
\hline blue-collar worker & 18 & 315,798 & 0.57 & 29 & 353,016 & 0.82 & 1.42 & $(0.79,2.57)$ & 1.69 & $(0.93,3.09)$ & \\
\hline fisher & 14 & 169,961 & 0.82 & 11 & 161,960 & 0.68 & 0.84 & $(0.38,1.84)$ & 1.03 & $(0.46,2.32)$ & \\
\hline others & 5 & 148,751 & 0.34 & 7 & 146,449 & 0.48 & 1.41 & $(0.45,4.46)$ & 2.13 & $(0.64,7.05)$ & \\
\hline $\begin{array}{l}\text { Comorbidities } \\
\text { hypertension }\end{array}$ & & & & & & & & & & & 0.15 \\
\hline No & 53 & $1,269,090$ & 0.42 & 99 & $1,445,802$ & 0.68 & 1.65 & $(1.18,2.30)^{* *}$ & 1.77 & $(1.26,2.47)^{* * *}$ & \\
\hline Yes & 24 & 133,909 & 1.79 & 13 & 75,798 & 1.72 & 0.92 & $(0.47,1.82)$ & 1.13 & $(0.57,2.26)$ & \\
\hline diabetes & & & & & & & & & & & 0.18 \\
\hline No & 62 & $1,335,540$ & 0.46 & 104 & $1,477,765$ & 0.7 & 1.52 & $(1.11,2.08)^{* *}$ & 1.72 & $(1.25,2.37)^{* * *}$ & \\
\hline Yes & 15 & 67,459 & 2.22 & 8 & 43,835 & 1.83 & 0.83 & $(0.35,1.97)$ & 0.95 & $(0.4,2.28)$ & \\
\hline hyperlipidemia & & & & & & & & & & & 0.20 \\
\hline No & 72 & $1,371,739$ & 0.52 & 111 & $1,503,288$ & 0.74 & 1.41 & $(1.05,1.90) *$ & 1.65 & $(1.22,2.23)^{* *}$ & \\
\hline Yes & 5 & 31,260 & 1.6 & 1 & 18,312 & 0.55 & 0.36 & $(0.04,3.10)$ & 0.49 & $(0.06,4.31)$ & \\
\hline CAD & & & & & & & & & & & 0.48 \\
\hline No & 65 & $1,357,680$ & 0.48 & 106 & $1,499,487$ & 0.71 & 1.48 & $(1.09,2.02) *$ & 1.68 & $(1.23,2.3)^{* *}$ & \\
\hline Yes & 12 & 45,319 & 2.65 & 6 & 22,113 & 2.71 & 0.98 & $(0.37,2.63)$ & 1.09 & $(0.4,2.97)$ & \\
\hline
\end{tabular}


Table 4. Cont.

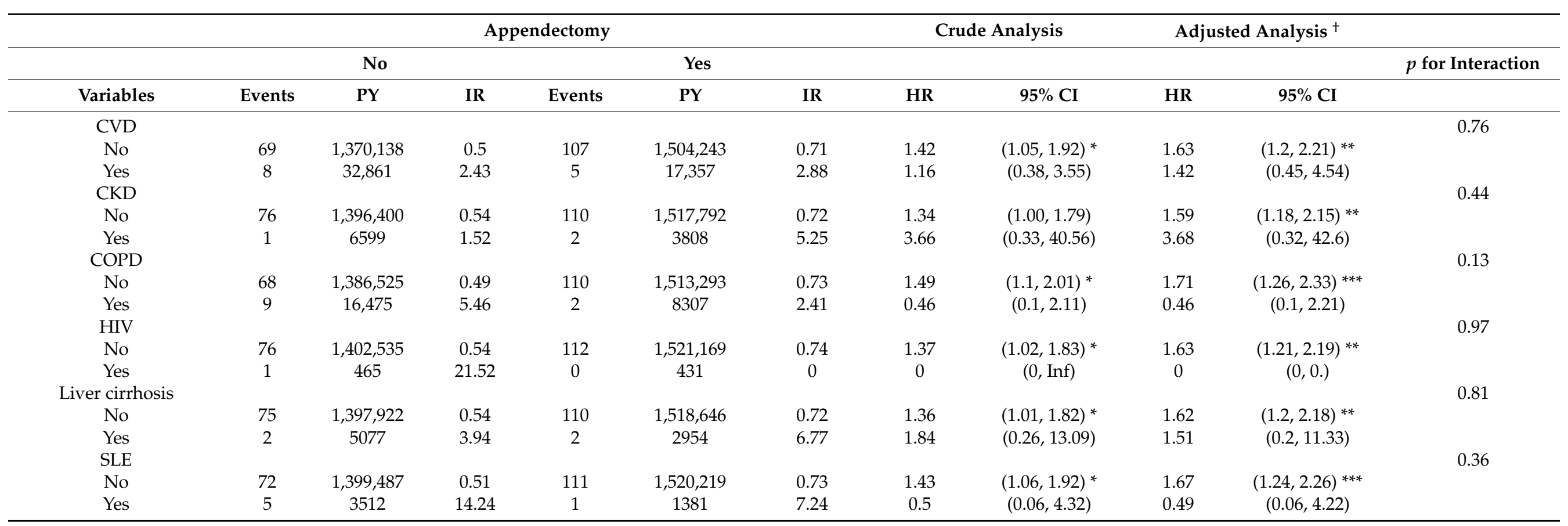

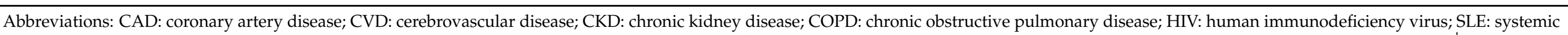

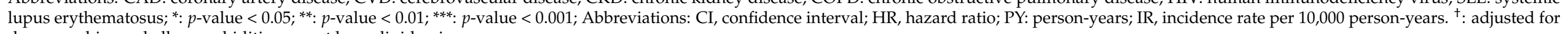
demographics and all comorbidities except hyperlipidemia. 
The alternative exposure (diverticulitis) showed no significant association between diverticulitis and subsequent hospitalizations for NTS (adjusted HR, 0.85; 95\% CI, 0.183.95) (Table 6). The cumulative incidence curve of NTS in the appendectomy cohort was significantly higher than that in the non-appendectomy group (log-rank test $p$-value $=0.04$ ) (Figure 1).

Table 5. Incidence and hazard ratios for NTS infection according to follow-up year.

\begin{tabular}{|c|c|c|c|c|c|c|c|c|c|c|}
\hline \multirow[b]{3}{*}{ Follow up Time } & \multicolumn{6}{|c|}{ Appendectomy } & \multicolumn{2}{|c|}{ Crude Analysis } & \multicolumn{2}{|c|}{ Adjusted Analysis ${ }^{\dagger}$} \\
\hline & & No & & & Yes & & & & & \\
\hline & Events & PY & IR & Events & PY & IR & HR & $95 \% \mathrm{CI}$ & HR & $95 \% \mathrm{CI}$ \\
\hline$<6$ months & 8 & 103,881 & 0.77 & 12 & 103,674 & 1.16 & 1.50 & $(0.61,3.68)$ & 1.83 & $(0.74,4.53)$ \\
\hline 6 months- 1 year & 11 & 103,081 & 1.07 & 6 & 102,896 & 0.58 & 0.55 & $(0.20,1.48)$ & 0.68 & $(0.25,1.89)$ \\
\hline$>1$ year & 58 & $1,196,037$ & 0.48 & 94 & $1,315,030$ & 0.71 & 1.47 & $(1.06,2.05) *$ & 1.74 & $(1.25,2.43) * *$ \\
\hline
\end{tabular}

*: $p$-value < 0.05; **: $p$-value < 0.01; Abbreviations: CI, confidence interval; HR, hazard ratio; PY: person-years; IR, incidence rate per 10,000 person-years. ${ }^{\dagger}$ : adjusted for demographics and all comorbidities except hyperlipidemia.

Table 6. Negative Control Exposure-Incidence Rate and Hazard Ratio.

\begin{tabular}{|c|c|c|c|c|c|c|c|}
\hline & & NTS & & & & & \\
\hline Variables & Events & PY & IR & cHR & $95 \%$ CI & aHR & $95 \% \mathrm{CI}^{\dagger}$ \\
\hline \multicolumn{8}{|l|}{ Diverticulitis } \\
\hline No & 4 & 14,099 & 2.84 & 1.00 & - & 1.00 & - \\
\hline Yes & 3 & 13,858 & 2.16 & 0.78 & $(0.17,3.47)$ & 0.85 & $\begin{array}{l}(0.18, \\
3.95)\end{array}$ \\
\hline
\end{tabular}

${ }^{\dagger}$ : adjusted for demographics and all comorbidities except hyperlipidemia.

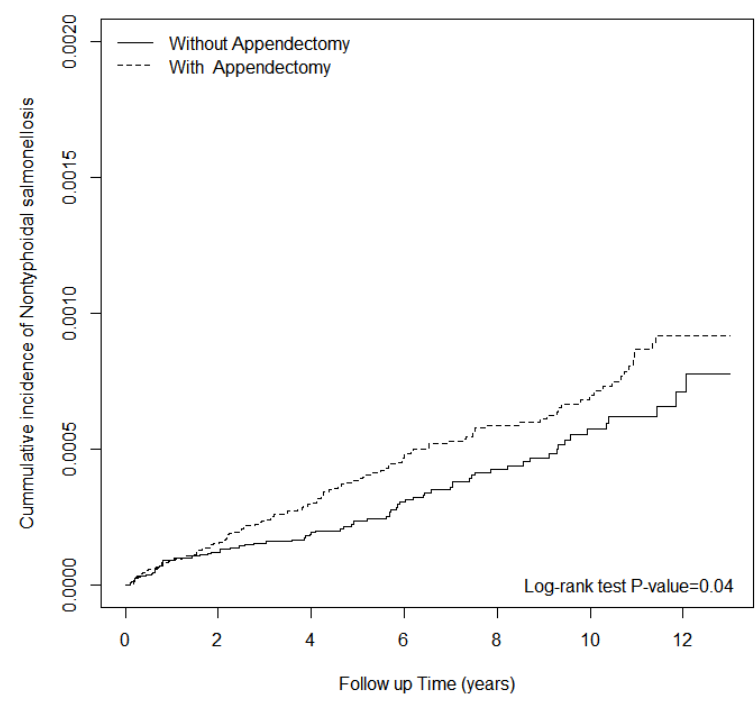

Figure 1. The cumulative incidence of hospitalized NTS infection for patients with and without appendectomy.

\section{Discussion}

In this study, a prior appendectomy was associated with a $61 \%$ increase of risk of developing hospitalized NTS. This is a novel finding. The link between previous appendectomy and subsequent NTS infection requiring hospitalization has never been discussed or confirmed before. Acute appendicitis occurs predominantly at 20 to 30 years of age with male predominance [28]. Similarly, our study found that about sixty percent of patients receiving an appendectomy were aged $<40$ years, with a male predominance. Some studies denoted that there might be postoperative change of the microbiome. Change of microbial composition was observed in patients received cholecystectomy [29,30]. Gastrointestinal 
microbiota showed higher species diversity and richness after gastrectomy in patients with gastric cancer [31]. Some studies have further accessed the interaction between dietary intake, gastric bypass surgery, and the trend of microbial change [32]. The balance of intestinal microbiota is critical to support the resistance against colonization by exogenous microorganisms. NTS was found to be competitive against the microbiome during inflammation in the gut and subsided when the inflammation ceased [33]. Butyrate as a feed additive has been widely used to improve the intestinal health of poultry and reduce the proliferation of Salmonella [34]. Appendectomy was reported significantly associated with low levels of butyrate-producing bacteria [35]. Furthermore, in one recent study, authors found that patients who underwent prophylactic appendectomy had lower levels of abundance and diversity of normal gastrointestinal tract species over the long-term [6]. Our findings are in alignment that the relative risk of NTS infections rise to statistically significant after one year.

A previous study demonstrated that risk factors for NTS infection consist of aging and immunocompromise [17], which corresponded with our findings in Table 2 (e.g., diabetes, COPD, liver cirrhosis, SLE, HIV).

The underlying mechanism by which appendectomy is associated with the risk of developing NTS infection remains unclear. First, the appendix contains large amounts of gut-associated lymphoid tissue, which is thought to be involved in immune function. Peyer's patches, and the appendix, are the sites of antigen sampling and induction in the mucosal immune system [36]. Therefore, an appendectomy might change the immune system. Secondly, the appendix can provide a suitable environment for normal intestinal flora through biofilm formation $[37,38]$. As a result, an appendectomy may disrupt the gut microbiota configurations subsequently supporting NTS development $[39,40]$.

The post hoc stratified analysis showed that compared with matched non-appendectomy controls, patients who received an appendectomy were associated with an increased risk of NTS especially in the subgroup of females and the subgroup for individuals aged 18 to 30 years. A recent meta-analysis of the global burden of invasive NTS disease did not find a link between sex and the incidence of invasive NTS disease [41]. It is intriguing however that this might not hold true in the context of prior appendectomy. Some animal and human studies have shown that disease patterns and gut microbiota differ by sex [42,43]. We speculate this novel result might be multifactorial, including environmental exposure (females are the main food handlers). However, further studies are needed to examine such discrepancies. Since advanced age is an independent risk factor for NTS infection, we specifically examined the interaction of age between appendectomy and outcome of interest in this study. In the age-subgroup analysis, compared with non-appendectomy controls, the population who received an appendectomy was at risk of new-onset NTS infection at the age of 18-30. The lack of association between appendectomy and NTS infection in the patients $>50$ years hold true in the elderly patients shown in Table 4 . New-onset post-appendectomy-associated NTS infection was higher in patients without underlying diseases. It may be possible to avoid hospitalized NTS in post-appendectomy patients in these subgroups.

Previous literature had described the advantages of using NHIRD in research [44]. These included enormous samples, one single ethnic population, and long-term comprehensive follow-up. We attempted to control the measurable covariates in both groups through PSM. In this study, we have examined and shown that diabetes, COPD, liver cirrhosis, SLE, and HIV infection are highly associated with NTS infection, and this is a kind of positive control analysis indicating the fitness of our models.

Some limitations in this study should be addressed. First, the diagnoses of NTS infection were based on administrative ICD-9-CM codes rather than a bacterial culture. The Bureau of NHI had a regular auditing mechanism. Quarterly expert reviews on random samples of inpatient claims data with a sampling rate of 1 in 10 were performed by the Bureau of NHI to ensure the accuracy. Misclassification bias may have occurred and some of the subgroup analyses where very few events were included may not be 
relevant. Second, the NHI program began in 1995; medical utilization before 1995 could not be traced. Therefore, the possibility that patients selected in the comparison cohort had undergone surgery before 1995 cannot be completely excluded. However, such a sampling bias would, on the contrary, underestimate the risk of the primary outcome [45]. Third, NHIRD does not provide lifestyle information, such as tobacco use, physical activity, body mass index, diet, and exercise. We have carefully used diabetes, hypertension, and hyperlipidemia as a proxy of metabolic status and COPD for tobacco use. Fourth, there is no detailed information about the route of NTS infection, its specific serotype, and level of disease severity in NHIRD and this is an inherent major limitation. In this study, we recruited patients from the subgroup of hospitalized NHIRD as a proxy for alluding to severe NTS infection.

Despite meticulous statistical analyses for possible confounding factor adjustment, bias may have occurred. We have applied a number of sensitivity analyses to control the measurable confounders and negative exposure controls to examine the unmeasured confounding. These observations suggest that the presence of confounding factors is less likely when assessed from this perspective. Finally, microbial dysbiosis may be a key intermediate between appendectomy and subsequent NTS infection, but that has not been established in the current study for the lack of detailed information regarding the interactions between appendectomy, change of microbiome metabolites (short-chain fatty acids, such as acetate, propionate, and butyrate), and the information of antibiotic use.

\section{Conclusions}

We conclude that Taiwanese residents with a history of appendectomy were associated with a risk of hospitalization for NTS. The risk was significant in women, and individuals aged 18-30 years. A small number of NTS infection diagnoses occurred in the study, thus limiting the conclusions somewhat. Clinicians are advised to implement prudently the post-operation education for patients to get rid of possible NTS contaminated food in the endemic area. It is of note that since this observation study was performed in one, relatively small country, if similar studies were to be done in the future in other countries of non-Asian origin, the results may be exactly the opposite.

Author Contributions: Study conception and design: D.-K.W., K.-S.Y., R.C., Y.-M.H., C.-H.H. Acquisition of data: H.-T.Y. Analysis and interpretation of data: D.-K.W., K.-S.Y., R.C., Y.-M.H., C.-H.H., J.C.-C.W. Validation: Y.-M.H., H.-T.Y. Writing (original draft preparation): K.-S.Y., D.-K.W. Writing (review and editing): R.C., Y.-M.H., C.-H.H. All authors have read and agreed to the published version of the manuscript.

Funding: This research received no external funding.

Institutional Review Board Statement: The study was conducted according to the guidelines of the Declaration of Helsinki. The study was approved by the Institutional Review Board of China Medical University Hospital Research Ethics Committee (CMUH104-REC2-115(AR-4)).

Informed Consent Statement: Patient consent was waived because the data were used anonymously and, in accordance with Personal Information Protection Act; the identities of individuals with insurance were recoded before analysis.

Data Availability Statement: Data are available from the National Health Insurance Research Database (NHIRD) published by Taiwan National Health Insurance (NHI) Bureau. Due to legal restrictions imposed by the government of Taiwan in relation to the "Personal Information Protection Act", data cannot be made publicly available.

Acknowledgments: The authors expressed their appreciation to the Department of Medical Education and Research and Research Center of Medical Informatics in Kaohsiung Veterans General Hospital for the comments. The authors also expressed appreciation to the Department of Medical Education and Research and Research Center of Medical Informatics in Kaohsiung Municipal United Hospital for the comments.

Conflicts of Interest: The authors declare no conflict of interest. 


\section{References}

1. Ferris, M.; Quan, S.; Kaplan, B.S.; Molodecky, N.; Ball, C.G.; Chernoff, G.W.; Bhala, N.; Ghosh, S.; Dixon, E.; Ng, S.; et al. The Global Incidence of Appendicitis: A Systematic Review of Population-based Studies. Ann. Surg. 2017, 266, 237-241. [CrossRef]

2. Lin, K.B.; Lai, K.R.; Yang, N.P.; Chan, C.L.; Liu, Y.H.; Pan, R.H.; Huang, C.H. Epidemiology and socioeconomic features of appendicitis in Taiwan: A 12-year population-based study. World J. Emerg. Surg. 2015, 10, 42. [CrossRef] [PubMed]

3. Girard-Madoux, M.J.; de Agüero, M.G.; Ganal-Vonarburg, S.C.; Mooser, C.; Belz, G.T.; Macpherson, A.J.; Vivier, E. The immunological functions of the Appendix: An example of redundancy? Semin. Immunol. 2018, 36, 31-44. [CrossRef] [PubMed]

4. Kooij, I.A.; Sahami, S.; Meijer, S.L.; Buskens, C.J.; Te Velde, A.A. The immunology of the vermiform appendix: A review of the literature. Clin. Exp. Immunol. 2016, 186, 1-9. [CrossRef]

5. Im, G.Y.; Modayil, R.J.; Lin, C.T.; Geier, S.J.; Katz, D.S.; Feuerman, M.; Grendell, J.H. The appendix may protect against Clostridium difficile recurrence. Clin. Gastroenterol. Hepatol. Off. Clin. Pract. J. Am. Gastroenterol. Assoc. 2011, 9, 1072-1077. [CrossRef] [PubMed]

6. Sánchez-Alcoholado, L.; Fernández-García, J.C.; Gutiérrez-Repiso, C.; Bernal-López, M.R.; Ocaña-Wilhelmi, L.; García-Fuentes, E.; Moreno-Indias, I.; Tinahones, F.J. Incidental Prophylactic Appendectomy Is Associated with a Profound Microbial Dysbiosis in the Long-Term. Microorganisms 2020, 8, 609. [CrossRef] [PubMed]

7. Lamarche, D.; Johnstone, J.; Zytaruk, N.; Clarke, F.; Hand, L.; Loukov, D.; Szamosi, J.C.; Rossi, L.; Schenck, L.P.; Verschoor, C.P.; et al. Microbial dysbiosis and mortality during mechanical ventilation: A prospective observational study. Respir. Res. 2018, 19, 245. [CrossRef] [PubMed]

8. Lai, S.W.; Lin, C.L.; Liao, K.F.; Tsai, S.M. Increased risk of pulmonary tuberculosis among patients with appendectomy in Taiwan. Eur. J. Clin. Microbiol. Infect. Dis. Off. Publ. Eur. Soc. Clin. Microbiol. 2014, 33, 1573-1577. [CrossRef]

9. Wu, M.C.; Tsou, H.K.; Lin, C.L.; Wei, J.C. Incidence and risk of sepsis following appendectomy: A nationwide population-based cohort study. Sci. Rep. 2020, 10, 10171. [CrossRef]

10. World Health Organization. Salmonella (Non-Typhoidal). Available online: https://www.who.int/news-room/fact-sheets/ detail/salmonella-(non-typhoidal) (accessed on 25 September 2020).

11. Wu, H.H.; Chen, Y.T.; Shih, C.J.; Lee, Y.T.; Kuo, S.C.; Chen, T.L. Association between recent use of proton pump inhibitors and nontyphoid salmonellosis: A nested case-control study. Clin. Infect. Dis. 2014, 59, 1554-1558. [CrossRef]

12. Steffen, R.; Hill, D.R.; DuPont, H.L. Traveler's diarrhea: A clinical review. JAMA 2015, 313, 71-80. [CrossRef] [PubMed]

13. Marzel, A.; Desai, P.T.; Goren, A.; Schorr, Y.I.; Nissan, I.; Porwollik, S.; Valinsky, L.; McClelland, M.; Rahav, G.; Gal-Mor, O. Persistent Infections by Nontyphoidal Salmonella in Humans: Epidemiology and Genetics. Clin. Infect. Dis. 2016, 62, 879-886. [CrossRef] [PubMed]

14. Haselbeck, A.H.; Panzner, U.; Im, J.; Baker, S.; Meyer, C.G.; Marks, F. Current perspectives on invasive nontyphoidal Salmonella disease. Curr. Opin. Infect. Dis. 2017, 30, 498-503. [CrossRef]

15. Katiyo, S.; Muller-Pebody, B.; Minaji, M.; Powell, D.; Johnson, A.P.; De Pinna, E.; Day, M.; Harris, R.; Godbole, G. Epidemiology and Outcomes of Nontyphoidal Salmonella Bacteremias from England, 2004 to 2015. J. Clin. Microbiol. 2019, 57, e01189-18. [CrossRef]

16. Parisi, A.; Crump, J.A.; Stafford, R.; Glass, K.; Howden, B.P.; Kirk, M.D. Increasing incidence of invasive nontyphoidal Salmonella infections in Queensland, Australia, 2007-2016. PLoS Negl. Trop. Dis. 2019, 13, e0007187. [CrossRef] [PubMed]

17. Gordon, M.A. Salmonella infections in immunocompromised adults. J. Infect. 2008, 56, 413-422. [CrossRef]

18. Kurtz, J.R.; Goggins, J.A.; McLachlan, J.B. Salmonella infection: Interplay between the bacteria and host immune system. Immunol. Lett. 2017, 190, 42-50. [CrossRef]

19. Lee, S.C.; Yang, P.H.; Shieh, W.B.; Lasserre, R. Bacteremia due to non-typhi Salmonella: Analysis of 64 cases and review. Clin. Infect. Dis. 1994, 19, 693-696. [CrossRef]

20. Shimoni, Z.; Pitlik, S.; Leibovici, L.; Samra, Z.; Konigsberger, H.; Drucker, M.; Agmon, V.; Ashkenazi, S.; Weinberger, M. Nontyphoid Salmonella bacteremia: Age-related differences in clinical presentation, bacteriology, and outcome. Clin. Infect. Dis. 1999, 28, 822-827. [CrossRef]

21. Hohmann, E.L. Nontyphoidal salmonellosis. Clin. Infect. Dis. 2001, 32, 263-269. [CrossRef]

22. Brown, M.; Eykyn, S.J. Non-typhoidal Salmonella bacteraemia without gastroenteritis: A marker of underlying immunosuppression. Review Of cases at St. Thomas' Hospital 1970-1999. J. Infect. 2000, 41, 256-259. [CrossRef] [PubMed]

23. Morpeth, S.C.; Ramadhani, H.O.; Crump, J.A. Invasive non-Typhi Salmonella disease in Africa. Clin. Infect. Dis. 2009, 49, 606-611. [CrossRef] [PubMed]

24. Kunz, L.J.; Waddell, W.R. Association of Salmonella enteritis with operations on the stomach. N. Engl. J. Med. 1956, 255, 555-559. [CrossRef] [PubMed]

25. Kolling, G.; Wu, M.; Guerrant, R.L. Enteric pathogens through life stages. Front. Cell. Infect. Microbiol. 2012, 2, 114. [CrossRef] [PubMed]

26. Wu, C.Y.; Kuo, K.N.; Wu, M.S.; Chen, Y.J.; Wang, C.B.; Lin, J.T. Early Helicobacter pylori eradication decreases risk of gastric cancer in patients with peptic ulcer disease. Gastroenterology 2009, 137, 1641-1648.e2. [CrossRef] [PubMed]

27. Wei, L.; Ratnayake, L.; Phillips, G.; McGuigan, C.C.; Morant, S.V.; Flynn, R.W.; Mackenzie, I.S.; MacDonald, T.M. Acid-suppression medications and bacterial gastroenteritis: A population-based cohort study. Br. J. Clin. Pharmacol. 2017, 83, 1298-1308. [CrossRef] [PubMed] 
28. Bhangu, A.; Søreide, K.; Di Saverio, S.; Assarsson, J.H.; Drake, F.T. Acute appendicitis: Modern understanding of pathogenesis, diagnosis, and management. Lancet 2015, 386, 1278-1287. [CrossRef]

29. Yoon, W.J.; Kim, H.N.; Park, E.; Ryu, S.; Chang, Y.; Shin, H.; Kim, H.L.; Yi, S.Y. The Impact of Cholecystectomy on the Gut Microbiota: A Case-Control Study. J. Clin. Med. 2019, 8, 79. [CrossRef]

30. Grigor'eva, I.N.; Romanova, T.I. Gallstone Disease and Microbiome. Microorganisms 2020, 8, 835. [CrossRef]

31. Erawijantari, P.P.; Mizutani, S.; Shiroma, H.; Shiba, S.; Nakajima, T.; Sakamoto, T.; Saito, Y.; Fukuda, S.; Yachida, S.; Yamada, T. Influence of gastrectomy for gastric cancer treatment on faecal microbiome and metabolome profiles. Gut 2020, 69, $1404-1415$. [CrossRef]

32. Crommen, S.; Mattes, A.; Simon, M.C. Microbial Adaptation Due to Gastric Bypass Surgery: The Nutritional Impact. Nutrients 2020, 12, 1199. [CrossRef] [PubMed]

33. Arguello, H.; Estelle, J.; Leonard, F.C.; Crispie, F.; Cotter, P.D.; O’Sullivan, O.; Lynch, H.; Walia, K.; Duffy, G.; Lawlor, P.G.; et al. Influence of the Intestinal Microbiota on Colonization Resistance to Salmonella and the Shedding Pattern of Naturally Exposed Pigs. mSystems 2019, 4, e00021-19. [CrossRef] [PubMed]

34. Onrust, L.; Baeyen, S.; Haesebrouck, F.; Ducatelle, R.; Van Immerseel, F. Effect of in feed administration of different butyrate formulations on Salmonella Enteritidis colonization and cecal microbiota in broilers. Vet. Res. 2020, 51, 56. [CrossRef] [PubMed]

35. Boesmans, L.; Valles-Colomer, M.; Wang, J.; Eeckhaut, V.; Falony, G.; Ducatelle, R.; Van Immerseel, F.; Raes, J.; Verbeke, K. Butyrate Producers as Potential Next-Generation Probiotics: Safety Assessment of the Administration of Butyricicoccus pullicaecorum to Healthy Volunteers. mSystems 2018, 3, e00094-18. [CrossRef] [PubMed]

36. Spahn, T.W.; Kucharzik, T. Modulating the intestinal immune system: The role of lymphotoxin and GALT organs. Gut 2004, 53, 456-465. [CrossRef]

37. Laurin, M.; Everett, M.L.; Parker, W. The cecal appendix: One more immune component with a function disturbed by postindustrial culture. Anat. Rec. 2011, 294, 567-579. [CrossRef]

38. Randal Bollinger, R.; Barbas, A.S.; Bush, E.L.; Lin, S.S.; Parker, W. Biofilms in the large bowel suggest an apparent function of the human vermiform appendix. J. Theor. Biol. 2007, 249, 826-831. [CrossRef] [PubMed]

39. Bazar, K.A.; Lee, P.Y.; Joon Yun, A. An "eye" in the gut: The appendix as a sentinel sensory organ of the immune intelligence network. Med. Hypotheses 2004, 63, 752-758. [CrossRef] [PubMed]

40. Barlow, A.; Muhleman, M.; Gielecki, J.; Matusz, P.; Tubbs, R.S.; Loukas, M. The vermiform appendix: A review. Clin. Anat. 2013, 26, 833-842. [CrossRef]

41. Stanaway, J.D.; Parisi, A.; Sarkar, K.; Blacker, B.F.; Reiner, R.C.; Hay, S.I.; Nixon, M.R.; Dolecek, C.; James, S.L.; Mokdad, A.H.; et al. The global burden of non-typhoidal salmonella invasive disease: A systematic analysis for the Global Burden of Disease Study 2017. Lancet Infect. Dis. 2019, 19, 1312-1324. [CrossRef]

42. Fairweather, D.; Frisancho-Kiss, S.; Rose, N.R. Sex differences in autoimmune disease from a pathological perspective. Am. J. Pathol. 2008, 173, 600-609. [CrossRef] [PubMed]

43. Kim, Y.S.; Unno, T.; Kim, B.Y.; Park, M.S. Sex Differences in Gut Microbiota. World J. Mens Health 2020, 38, 48-60. [CrossRef] [PubMed]

44. Hsing, A.W.; Ioannidis, J.P. Nationwide Population Science: Lessons from the Taiwan National Health Insurance Research Database. JAMA Intern. Med. 2015, 175, 1527-1529. [CrossRef] [PubMed]

45. Copeland, K.T.; Checkoway, H.; McMichael, A.J.; Holbrook, R.H. Bias due to misclassification in the estimation of relative risk. Am. J. Epidemiol. 1977, 105, 488-495. [CrossRef] [PubMed] 